\title{
Co-assembly, spatiotemporal control and morphogenesis of a hybrid protein-peptide system
}

\author{
Karla E. Inostroza-Brito, Estelle Collin, Orit Siton-Mendelson, Katherine H. Smith, Amàlia \\ Monge-Marcet, Daniela S. Ferreira, Raúl Pérez Rodríguez, Matilde Alonso, \\ José Carlos Rodríguez-Cabello, Rui L. Reis, Francesc Sagués, Lorenzo Botto, Ronit Bitton, \\ Helena S. Azevedo and Alvaro Mata
}

$\mathrm{N}$ ature uses self-assembly to create a widespread variety of complex structures with elaborate geometries and outstanding properties ${ }^{1}$ such as hierarchical order, adaptability, selfhealing and bioactivity. Developing new bioinspired processes based on dynamic self-assembly could facilitate the fabrication of synthetic three-dimensional (3D) materials with enhanced complexity, dynamic properties and functionality ${ }^{2}$. Proteins are particularly attractive building blocks because of their versatility and biofunctionality ${ }^{3}$. Elastin-like polypeptides (ELPs) ${ }^{4}$ are recombinant proteins that have generated great interest ${ }^{5}$ as a result of their modular structure, bioactivity, ease of design and production, and the possibility to create robust and elastic materials ${ }^{5,6}$. ELPs allow for a tunable molecular design ${ }^{7}$ and are based on the tropoelastin recurrent motif Val-Pro-Gly-X-Gly (VPGXG), in which $X$ is any amino acid other than proline ${ }^{7}$. This repeating pentapeptide provides ELPs with a thermoresponsive behaviour. Below a critical transition temperature $\left(T_{\mathrm{t}}\right)$, the ELP molecule undergoes a reversible-phase transition wherein the protein is soluble in aqueous solution and becomes highly solvated, surrounded by clatharate-like water structures. Above the $T_{\mathrm{t}}$, the hydrophobic domains dehydrate and the protein chain hydrophobically collapses and aggregates to form a phaseseparated state 8 .

The use of natural and synthetic proteins to create functional materials has been hindered by the difficulty in controlling their conformation and nanoscale assembly with the precision required to form macroscopic materials. This limitation has driven the development of simpler and more-predictable peptide-based materials ${ }^{9,10}$. Peptide amphiphiles (PAs), for example, are synthetic molecules that can self-assemble into nanofibres and create functional 3D hydrogels that emulate the fibrous architecture of the extracellular matrix $(\mathrm{ECM})^{11,12}$. Nonetheless, most peptide and/or protein materials are formed through equilibrium-based self-assembly approaches that are capable of generating stable supramolecular structures, but with limited hierarchy and spatiotemporal control, which has hindered their functionality ${ }^{2}$.

Novel approaches based on the dynamic self-assembly of inorganic building blocks ${ }^{13-15}$, actin self-organization ${ }^{16}$ and the combination of top-down processes with peptide self-assembly have been reported recently ${ }^{17}$. In particular, Stupp and co-workers have described a self-assembling membrane system obtained through strong electrostatic interactions between PAs and oppositely charged polysaccharides ${ }^{18}$. However, the possibility to exploit the unique structural and functional properties of proteins to create dynamic hierarchical materials remains an elusive target. In this study, we attempt to overcome this hurdle by using self-assembling peptides to promote protein conformational changes and guide their assembly into complex, yet functional, materials. We report the discovery and development of a protein/peptide system that can be maintained in non-equilibrium for substantial periods of time with a high spatiotemporal control over its assembly. The system permits the fabrication of robust membranes that exhibit a distinctive multilayered hierarchical architecture and a spectrum of dynamic properties, which include the capacity to undergo morphogenesis into tubular structures (tubes or networks of tubes). We demonstrate the potential of the system for fabricating geometrically complex and bioactive scaffolds. 


\begin{tabular}{|c|c|c|c|c|c|}
\hline ELPs & Sequence & $M_{\mathrm{W}}(\mathrm{kDa})^{*}$ & $\mathrm{p} l^{\dagger}$ & $\zeta(\mathrm{mV})^{\ddagger}$ & $T_{\mathrm{t}}\left({ }^{\circ} \mathrm{C}\right)^{*}$ \\
\hline ELP2 & MESLLP-[(VPGVG VPGVG VPGEG VPGVGVPGVG) $)_{10^{-}}(\text {VGIPG) })_{60}-\mathrm{V}$ & 46.9 & 3.2 & $-23.0 \pm 0.3$ & 19.5 \\
\hline ELP4 & MESLLP-[(VPGVG VPGVG VPGEG VPGVG VPGVG) $)_{10}-(\text { VGIPG)60 }]_{2}-\mathrm{V}$ & 93.2 & 2.9 & $-25.9 \pm 2.1$ & 19.8 \\
\hline ELP5 & MESLLP-[(VPGVG VPGVG VPGEG VPGVG VPGVG) $10^{-}(\text {VGIPG) } 60]_{2}-\left[(\mathrm{VPGIG})_{10^{-}}\right.$ & 112.3 & 3.4 & $-30.5 \pm 4.0$ & 19.3 \\
\hline
\end{tabular}

\begin{tabular}{|c|c|c|c|c|}
\hline PAs & Sequence & $M_{\mathrm{W}}(\mathrm{kDa})^{*}$ & $\mathrm{pl}^{*}$ & $\zeta(m V)^{\dagger}$ \\
\hline PAK2 & $\mathrm{C}_{15} \mathrm{H}_{31} \mathrm{CONH}-\mathrm{VVVAAAKK}-\mathrm{CONH}_{2}$ & 1.0224 & 10 & $67.8 \pm 5.2$ \\
\hline PAK3 & $\mathrm{C}_{15} \mathrm{H}_{31} \mathrm{CONH}-\mathrm{V} V$ VAAKKK-CONH ${ }_{2}$ & 1.1506 & 10.3 & $67.5 \pm 3.3$ \\
\hline PAK4 & $\mathrm{C}_{15} \mathrm{H}_{31}$ CONH-VVVAAAKKKK-CONH 2 & 1.2788 & 10.5 & $66.6 \pm 4.0$ \\
\hline $\mathrm{Cl} 6 \mathrm{~K} 3$ & $\mathrm{C}_{15} \mathrm{H}_{31} \mathrm{CONH}-\mathrm{KKK}-\mathrm{CONH} \mathrm{H}_{2}$ & 0.6396 & 10.3 & $40.9 \pm 3.1$ \\
\hline PAE3 & $\mathrm{C}_{15} \mathrm{H}_{31}$ CONH-VVAAAEEE-CONH ${ }_{2}$ & 1.1531 & 3.7 & $-33.1 \pm 3.0$ \\
\hline
\end{tabular}

\section{Results and discussion}

Protein and peptide building blocks. The protein/peptide system is formed by the co-assembly of an ELP (ELP5) and a PA (PAK3). The ELP5 sequence (Table 1) used is a long pentablock molecule that contains distinct hydrophobic blocks, which allows coacervation and a highly collapsed conformation in an aqueous environment above its $T_{\mathrm{t}}$ of $19{ }^{\circ} \mathrm{C}$ (Methods). It is negatively charged above its isoelectric point of 3.4 (Table 1 and Supplementary Fig. 1). Four of its blocks are based on the elastin sequence VPGXG, with X being isoleucine (I), valine (V) or glutamic acid (E). The fifth block contains an Arg-Gly-Asp-Ser (RGDS) motif to promote cell adhesion. The PAK3 (Table 2) is a nine amino acid peptide attached to an alkyl tail of 16 carbons capable of self-assembling into nanofibres when the charges of its peptide segment are screened $^{18,19}$. It was found positively charged below its isoelectric point of 10.3 (Table 2 and Supplementary Fig. 1). Additional ELP and PA molecules were synthesized to help elucidate the assembly mechanism (Tables 1 and 2).

Material morphogenesis into functional tubular structures. When a PAK3 aqueous solution $(8.7 \mathrm{mM})$ is immersed in a large volume of an ELP5 aqueous solution (0.1 mM) above the ELP5's $T_{t}$, a dynamic interfacial assembly spontaneously develops (Fig. 1a). In this way, a closed membrane is formed that entraps the PA solution inside it and leaves the ELP solution outside. Self-assembled membranes formed at the interface between a solution of linear polyelectrolytes and a solution of either oppositely charged PAs ${ }^{18-22}$ or surfactant molecules ${ }^{23}$ have been reported. However, in our system, within the first minute of formation and on contact with any surface, the membrane spontaneously, yet controllably, adheres, focally opens and seals to the surface (Fig. 1a-g). The process leads to the formation of a 3D tube as it keeps the two solutions separated (Fig. 1a and Supplementary Movie 2a). A major advantage of this system is the possibility to manipulate the tube in real time and with spatiotemporal control. A simple 1D extension promotes anisotropic growth (Fig. 1b,c and Supplementary Movie 2b). Additional manipulation of the tube, by modulating the number and size of the touching interfaces, generates a more-complex morphogenesis into a network of tubes (Fig. 1b,e,f and Supplementary Movie 2c,d). The system can repeatedly self-heal large-scale ruptures without the need for additional manipulation (Fig. 1g and Supplementary Movie 2d). After 48 hours, the tubular structure is robust and can be handled easily with tweezers both in water and air (Fig. $1 \mathrm{~h}$ and Supplementary Movie 2e). Scanning electron microscopy (SEM) revealed a nanofibrous multilayered membrane of about $30 \mu \mathrm{m}$ in thickness (Fig. 1i). The outer surface of the membrane is highly adhesive in comparison with its inner surface. Confocal microscopy (Fig. 2a) and time-offlight secondary-ion mass spectrometry (TOF-SIMS) analysis (Fig. $2 b$ and Supplementary Section 3) confirmed this anisotropy, and revealed a higher prevalence of ELP5 on the outer surface with the inner surface richer in PAK3.

Mechanisms of molecular and hierarchical assembly ELP-PA interactions. Strong electrostatic forces have been shown to drive the assembly of interfacial systems ${ }^{18,22,23}$. Zeta potential ( $\zeta$ ) measurements demonstrated that the ELP5 and PAK3 need to be oppositely charged to co-assemble into the dynamic system (Fig. 2c). A lack of visible interaction when combining the ELP5 with a negatively charged PA (PAE3 (Supplementary Figs 4 and 5)) indicates that the initial electrostatic complexation is necessary. However, robust membranes with the distinctive dynamic properties are obtained when $\zeta$ of one molecule is marginally opposite that of the other (Fig. 2c). Outside this window, the membrane is either static (does not undergo morphogenesis) or dynamic, but unstable. To investigate further the role of the electrostatic interactions, PA molecules with lower (PAK2) or higher (PAK4) charge densities were combined with the ELP5. ELP5/PAK4 systems were similar to those made with PAK3 but exhibited a weaker and looser membrane (Supplementary Fig. 6). In contrast, ELP5/PAK2 systems led to static membranes that were thinner, transparent, tended to shrink in time and did not exhibit the dynamic behaviours of ELP5/PAK3 (Fig. 3e,f and Supplementary Section 7). Differences in both the assembly mechanism and the architecture of the resulting membrane were evidenced by small-angle X-ray scattering (SAXS) analysis and SEM observations, respectively (Fig. 3a,b,d).

ELP molecules above the $T_{\mathrm{t}}$ undergo a conformational change because of dehydration of their hydrophobic domains ${ }^{24}$. To investigate the role of hydrophobic interactions in our system, turbidity and dynamic light scattering (DLS) analyses above and below the $T_{\mathrm{t}}$ were performed. The turbidity profile of $0.008 \mathrm{mM}$ ELP5 and $0.174 \mathrm{mM}$ PAK3 solutions measured at a $300 \mathrm{~nm}$ revealed that a significant aggregation is obtained only when molecules are combined above the $T_{\mathrm{t}}$ (Fig. $2 \mathrm{~d}$ ). Similarly, DLS measurements demonstrated that a solution of a 


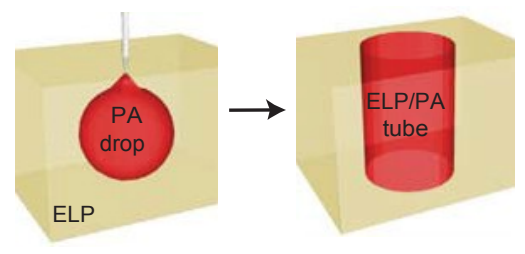

b

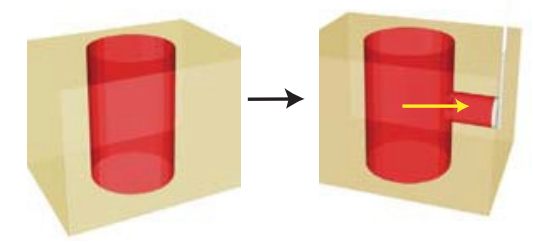

c

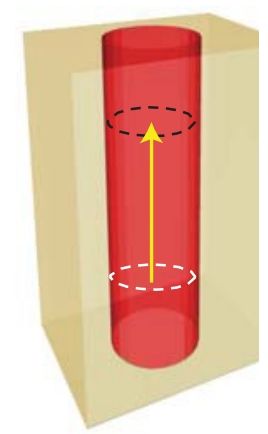

g

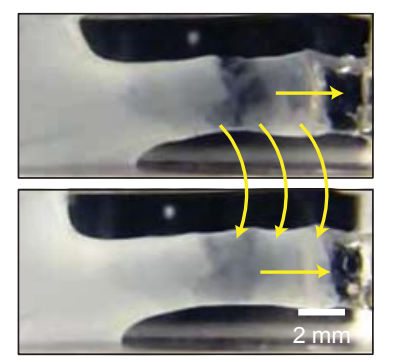

Longitudinal growth
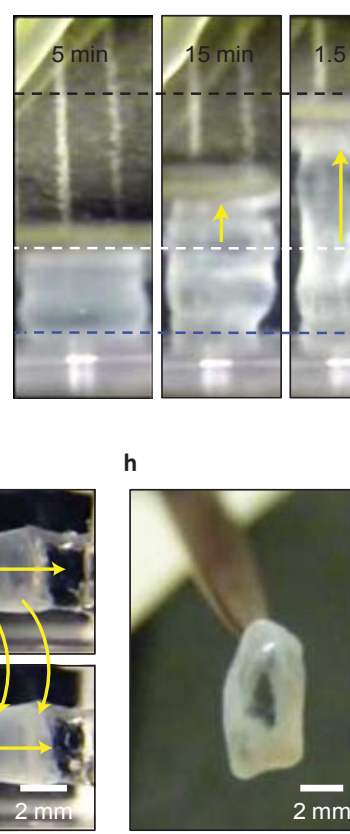
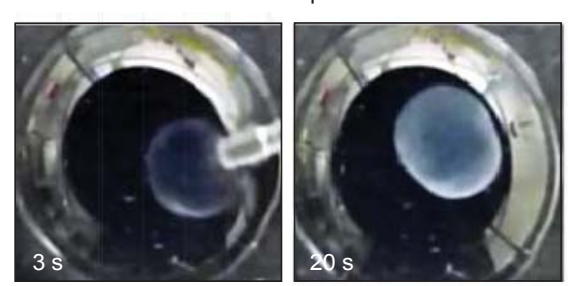
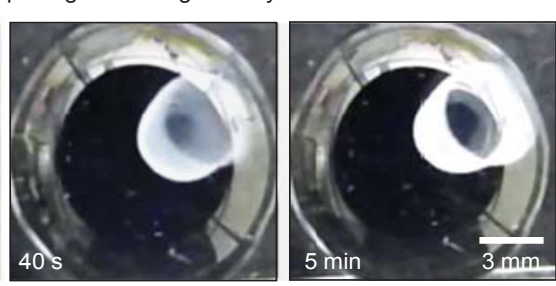

Anisotropic growth of lateral tube

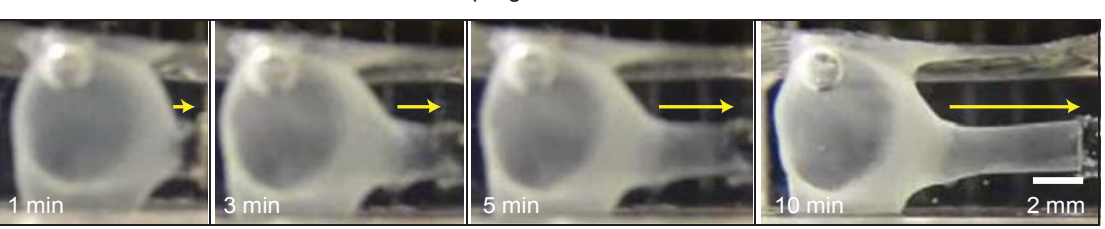

d

Four-way tubes

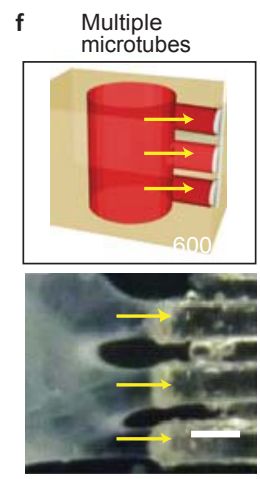

i

Figure I | Self-assembly, dynamic properties and architecture of the hybrid ELP5/PAK3 system. a, Time-lapse images of the self-assembly of a closed ELP5/PAK3 membrane on immersion of a PAK3 solution inside a larger volume of ELP5 solution, and the controlled disassembly of the membrane on touching the bottom surface and the air/water interface (Supplementary Movie 2a). b,c, The system permits a directed self-assembly into robust tubes that can be grown on demand by simply touching and displacing the membrane (b) (Supplementary Movie 2c), or by adding more ELP5 solution

(c) (Supplementary Movie 2b). d-f, Morphogenesis can be controlled by regulating the size and number of surfaces that touch the membrane, which enables the formation of half-sphere structures (d), four-way tubes (e) and tubular networks with multiple tubes of different sizes (f). g,h, The system permits repeated self-healing of large-scale ruptures within a few minutes and without requiring additional manipulation (g) (Supplementary Movie $2 \mathrm{~d}$ ), and is able to generate robust membranes with elastic behaviour that can be manipulated in air or water (h) (Supplementary Movie 2e). i, SEM images show the cross-section of an ELP5/PAK3 membrane that exhibits the distinctive nanofibrous multilayered architecture.

mixture of ELP5 and PAK3 molecules contained larger particles above the ELP5's $T_{\mathrm{t}}$ (Supplementary Fig. 8). Above the $T_{\mathrm{t}}$, the hydrophobic domains of the ELP5 are dehydrated ${ }^{4}$, which would result in the display of large hydrophobic domains in which the PAK3 molecules could interact through hydrophobic interactions and therefore result in a larger particle size and aggregation. In contrast, below the $T_{\mathrm{t}}$, the rehydration of the hydrophobic domains of the ELP5 would inhibit these hydrophobic interactions. We hypothesize that these interactions take place between the hydrophobic regions of both ELP5 and PAK3 molecules, as has been previously reported for interactions between proteins and surfactants ${ }^{25,26}$. To test this hypothesis, ELP5 and PAK3 solutions were mixed below the $T_{\mathrm{t}}\left(4{ }^{\circ} \mathrm{C}\right)$, which resulted in a disordered white viscous aggregate (Supplementary Fig. 9a). In addition, when a formed tube made above the ELP5's $T_{t}$ was immersed in a cold aqueous solution below the $T_{\mathrm{t}}\left(4^{\circ} \mathrm{C}\right)$ (Supplementary Fig. 9b,c), a significant weakening of the construct was observed, which demonstrates the need for the dehydrated hydrophobic domains of the ELP5. 

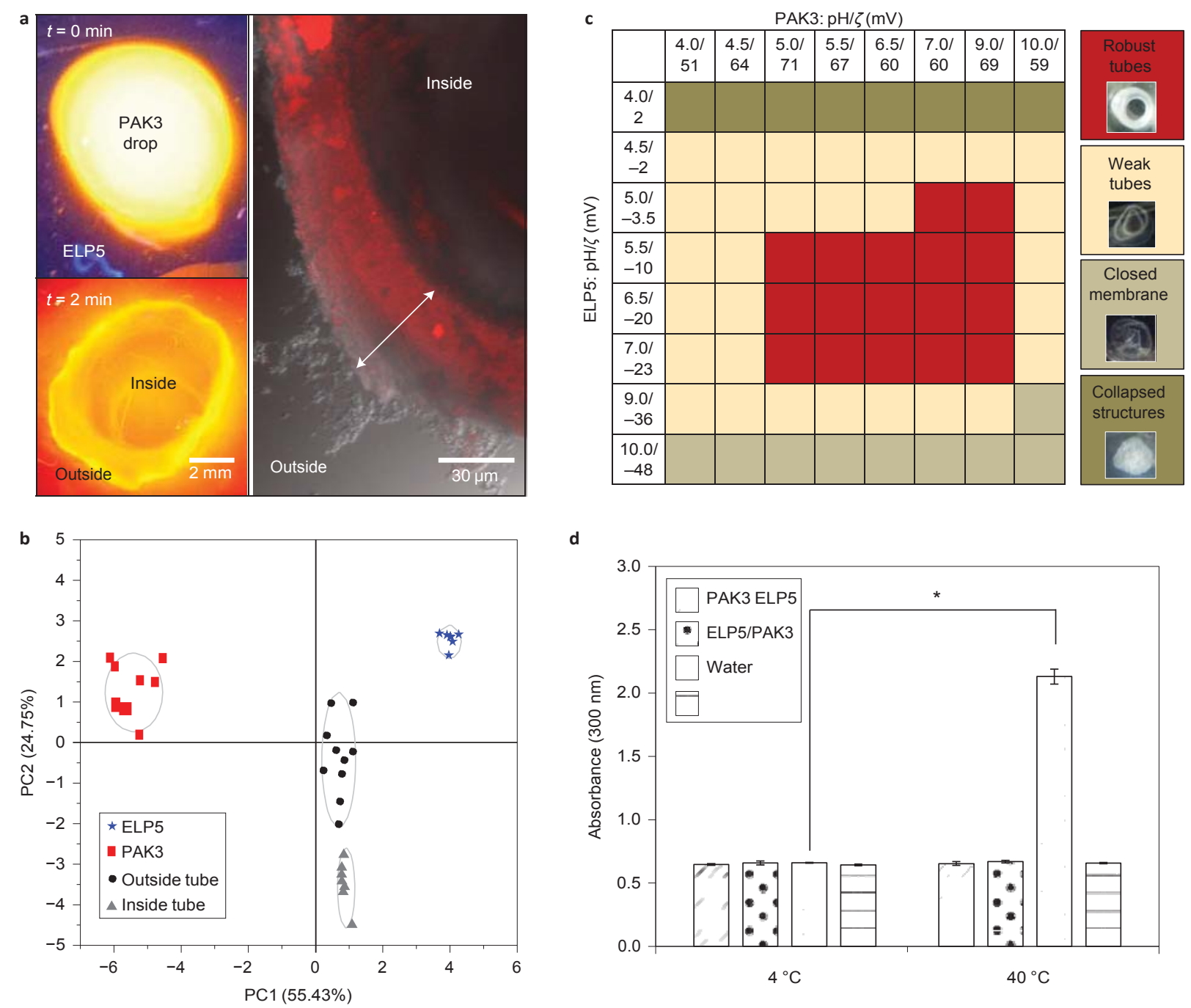

d

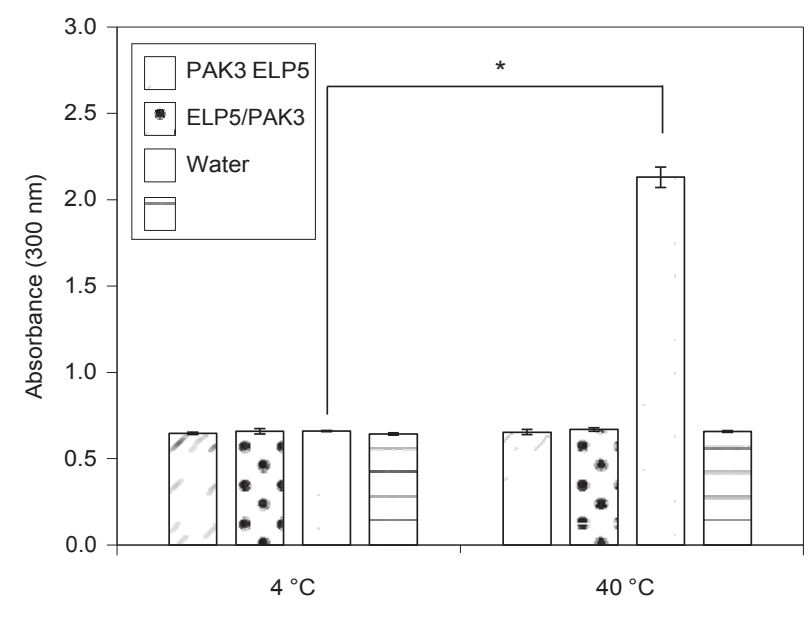

Figure 2 | Molecular interactions and composition of the ELP5/PAK3 membrane. a, Fluorescent images that demonstrate the diffusion of the fluorescently labelled PAK3 from the drop of PA towards the ELP5 solution during the self-assembly of the ELP5/PAK3 membrane, and a confocal microscopy image (right) of the membrane cross-section, which exhibits a higher concentration of PAK3 (red) on the inner surface of the membrane. b, Principal component (PC) loadings from the TOF-SIMS analysis confirm a larger presence of PAK3 on the inner surface of the membrane and a larger presence of ELP5 on the outer surface. c, The table illustrates the role of $\mathrm{pH}$ and $\zeta$ on the formation of the ELP5/PAK3 system and the optimum conditions (red) required to enable the dynamic properties of the system. $d$, Turbidity profile of solutions of PAK3 and ELP5 demonstrate the need for the self-assembly process to take place above the transition temperature of the ELP5 to generate the dynamic system and the presence of hydrophobic interactions between the two molecules. Error bars represent \pm s.d. for $n=9$. ${ }^{*} P<0.05$.

Molecular weight and structure. When combined with PAK3, all the ELP molecules (ELP2, ELP4 and ELP5), independent of molecular weight, generated membranes with dynamic properties. The mostrobust tubular structures were formed using ELP5 and the weakest with ELP2 (Fig. 3a). To study the role of the ELP5 molecular structure, the protein elastin, the neutral homopolymer polyethylene glycol (PEG) and polyacrylamide were also combined with all the PA molecules. Static closed membranes were formed when using the elastin, but no visible interaction was observed when using the PEG or polyacrylamide (Supplementary Section 10). These results demonstrate that the molecular weight and amino-acid sequence of the ELP affect both the dynamic behaviour and the structural integrity of the system.

Membrane formation and growth. On contact between the two solutions, the forming membrane acts as a diffusion barrier characterized by the low permeability to ELP5, across which the PAK3 can diffuse outwards driven by an osmotic pressure imbalance (Fig. 2a). The initial ELP5/PAK3 membrane assembles as the PAK3 molecules that cross the barrier interact with the ELP5 molecules present in the external solution. SAXS patterns of the ELP5/PAK3 membrane (Fig. 3b) exhibit a distinct peak at $q_{\max } \sim 0.023 \mathrm{~A}^{-1}$ ( $q$, scattering vector), indicative of a repeating distance of $\sim 27 \mathrm{~nm}\left(d=2 \pi / q_{\max }\right)$. This distance could correspond to the multilayers observed by SEM. The SAXS profile also shows a shoulder at $q_{\max } \sim 0.07 \mathrm{~A}^{-1}$ that can be attributed to the diameter of PAK3 fibres in the membrane (PAK3 fibre diameter $=9 \mathrm{~nm}$ as measured by cryo-transmission electron microscopy (TEM) (Fig. 3c) and SAXS (Supplementary Fig. 11a)). Replacing the ELP5 with ELP4 and PAK3 with PAK4 resulted in multilayered (Fig. 3a) and weaker membranes as observed on manipulation. Interestingly, no membranes with the multilayered architecture were formed when using PAK2 (that is, ELP2/PAK2, ELP4/PAK2 and ELP5/PAK2), but rather were structurally similar to the $\mathrm{PA} /$ polysaccharide system reported by Stupp and co-workers ${ }^{18,27}$, as observed by SEM (Fig. 3a) and SAXS (Fig. 3b) measurements. 

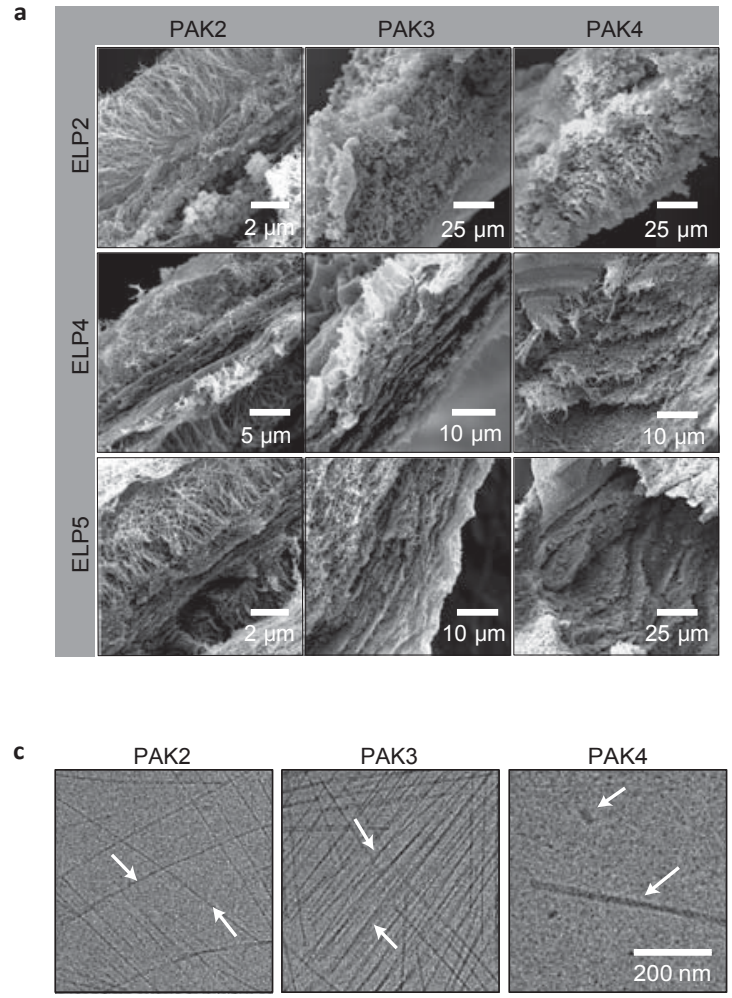
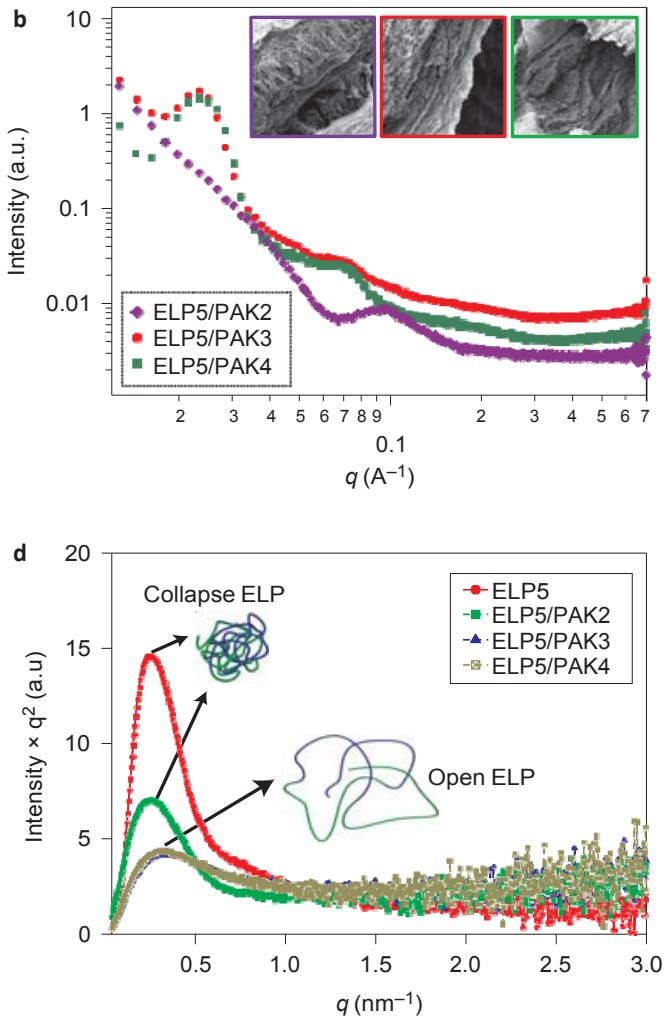
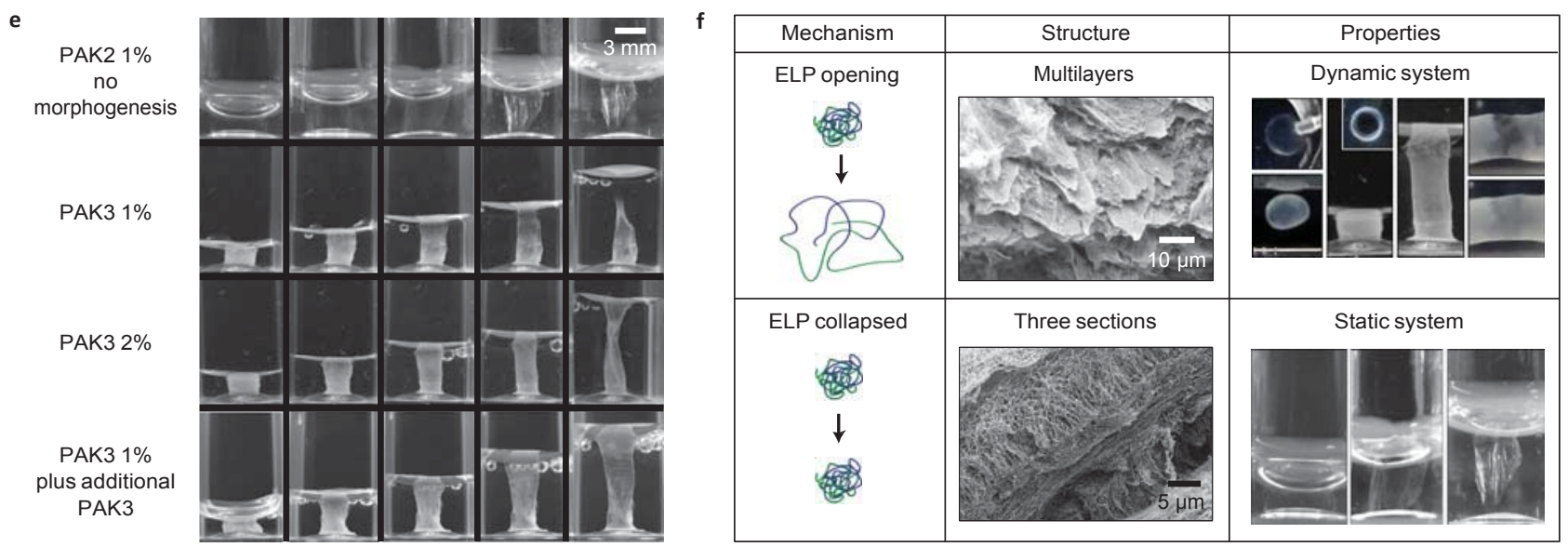

Figure 3 | Relation between the molecular mechanism, membrane architecture and properties of the ELP5/PAK3 system. a, The SEM images show the differences in membrane architecture depending on the sequence of the PA and ELP molecules used. b, Experimental SAXS data of membranes made from different ELP/PA combinations confirm the distinctive multilayered architecture of the ELP5/PAK3 (filled circles) and ELP5/PAK4 (filled squares) membranes compared with the ELP5/PAK2 (filled diamonds) membranes. c, Cryo-TEM images of PAK2, PAK3 and PAK4 confirm the presence of cylindrical micelles (white arrows) at the concentration used for SAXS. d, A Kratky plot of the SAXS scattering data shows that above the ELP5's Tt, PAK3 and PAK4 open the ELP5 molecules, but PAK2 does not. e, The time-lapse images demonstrate the static properties of ELP5/PAK2 membranes compared with the dynamic properties of ELP5/PAK3 membranes on the addition of ELP5 solution. Longer tubes are obtained with a higher concentration of PA or by injecting additional PAK3 solution inside a formed tube, which demonstrates that morphogenesis takes place because of the recruitment of ELPand PA molecules in solution. $\mathrm{f}$, Schematic that summarizes the relation between the molecular self-assembly mechanism, the resulting membrane architecture and the corresponding membrane properties. The ELP5/PAK3 system is characterized by the opening of the ELP5 molecules on self-assembly, which leads to the multilayered architecture of the membrane and its dynamic properties.

The different membrane structure and properties of ELPS/PAK2 systems are probably a result of a different molecular mechanism ${ }^{22,27}$ compared with that of the ELP5/PAK3 system. These results demonstrate that the presence of the distinctive multilayered architecture is required for the controlled dynamic behaviours observed in the ELP5/PAK3 system.
The role of the conformational change of ELP5, from collapsed to open, and its interaction with PAK3 was investigated. The studies were conducted at relatively lower molar concentrations (0.008 mM ELP and $0.174 \mathrm{mM} \mathrm{PA}$ ), but above the critical micelle concentration of PAK3 ${ }^{28}$. Cryo-TEM imaging of PAK2, PAK3 and PAK4 solutions at $0.174 \mathrm{mM}$ was performed to confirm that 
a Cells seeded inside and outside the tubes
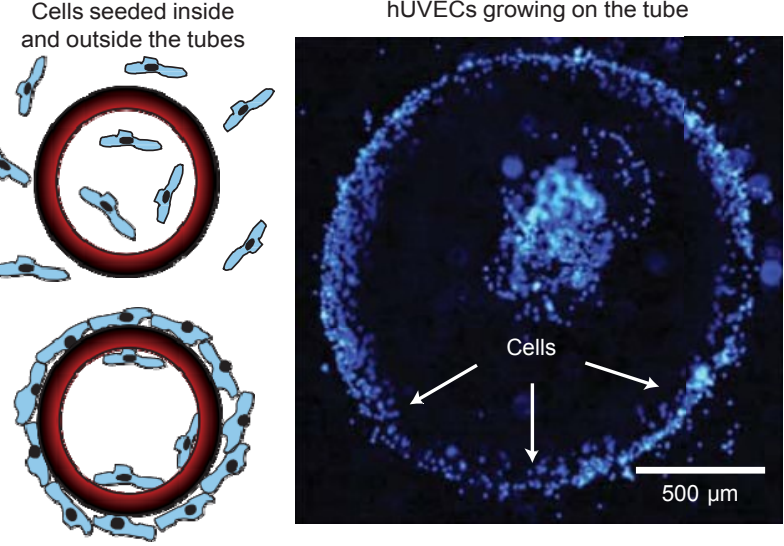

C

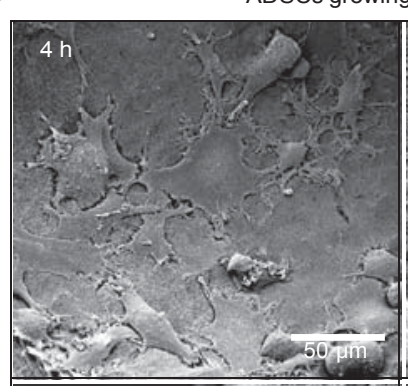

ADSCs growing outside the tube

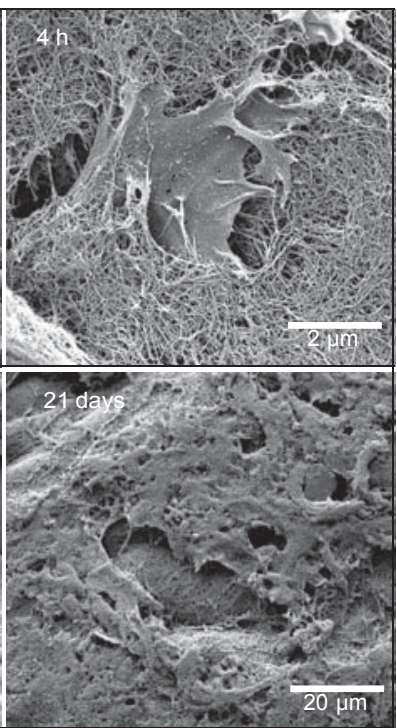

b

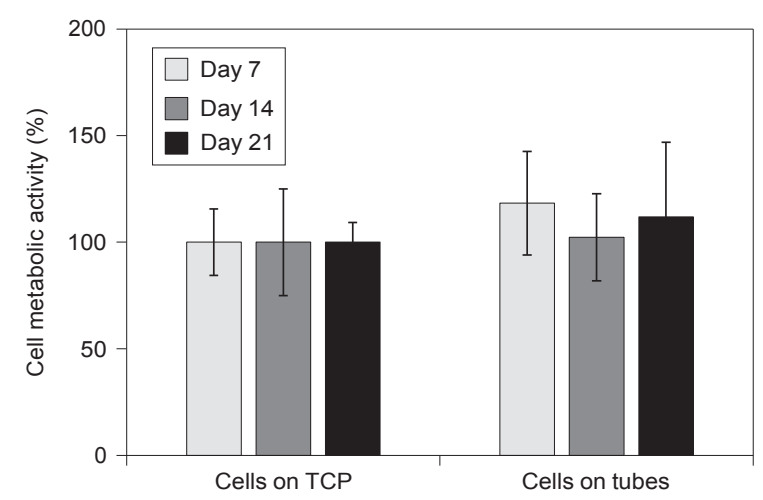

d

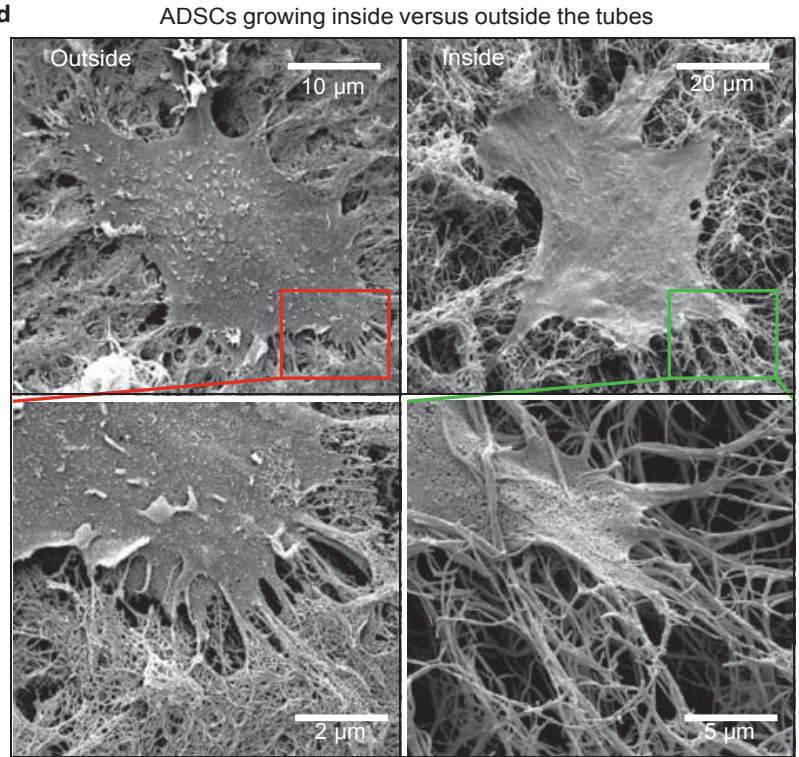

Figure 4 Application in tissue engineering. a, Schematic illustration of the seeding of cells inside and outside the tubes and a fluorescence microscopy image of DAPI (4,6-diamidino-2-phenylindole)-stained hUVECs growing on an ELP5/PAK3 tube after four hours of cell seeding. b, Cell metabolic activity was assessed by alamar blue staining on mADSCs. The results demonstrate that cell metabolic activity and viability on the tubes is at least similar to that of cells growing on TCP for up to 21 days. Error bars represent \pm s.d. for $n=9$. $* P<0.05$. c, SEM images obtained at different time points show the ability of the ELP5/PAK3 tubes to support mADSC colonization, growth and extracellular matrix production. d, SEM images of mADSCs growing on both sides of the ELP5/PAK3 tube at day 7. Cells on the outside of the tube were observed to be spread more and to interact extensively with the nanofibres compared with cells on the inside of the tube, which confirms the higher presence of the RGDS-containing ELP5 on the outside of the tube.

the PAs retained their cylindrical micelle morphology and their ability to form nanofibres at the concentrations used for SAXS experiments (Fig. 3c). The Kratky plot of the SAXS scattering data (Fig. 3d) shows that, above the $T_{\mathrm{t}}$, combinations of ELP5 (0.008 mM) and PAK2 (0.174 mM) yielded a prominent peak typical of globular 'folded' proteins, but both ELP5/PAK3 and ELP5/PAK4 solutions showed a Kratky plot typical of partially 'unfolded' proteins ${ }^{29}$. In contrast, the SAXS data of the ELP5/PAK3 solution below the $T_{\mathrm{t}}$ showed scattering curves that are typical of an extended molecule similar to that of ELPs below their $T_{\mathrm{t}}$ (that is, 'unfolded' proteins) (Supplementary Fig. 11b,c). It is known that globular proteins have the tendency to unfold when combined with ionic surfactants ${ }^{30,31}$. To test this hypothesis, a $0.008 \mathrm{mM}$ ELP5 solution was combined with the oppositely charged commercial surfactant dodecyltrimethylammonium bromide (DTAB) above its critical micelle concentration $(18 \mathrm{mM})$. DTAB did not alter the ELP5 conformation (Supplementary Fig. 11d). As for DTAB, the peptide C16K3 forms spherical micelles ${ }^{27}$. C16K3 (0.174 mM) was combined with ELP5 (0.008 mM) and, similar to DTAB, the interaction did not change the conformation of the ELP5 (Supplementary Fig. 11e), which suggests that the opening of ELP5 requires the presence of PA with the capability to self-assemble into fibres. These results confirm the critical role of the conformational opening of the ELP5 molecules in the presence of PAK3 molecules (Fig. 3f). Pattern formation is often associated with delicate coupling reaction-diffusion processes ${ }^{32}$, as illustrated, for example, by Turing's model $^{33}$. The regular multilayered architecture of the membrane may emerge from the nonlinearity of the underlying reaction-diffusion process that takes place during the ELP5/PAK3 interactions. Verification of this hypothesis is, of course, not trivial and requires further studies.

Tubular morphogenesis. We divide tubular morphogenesis into two stages: (1) opening of the membrane and (2) growth of the tubular structure. Within the first minute of the ELP5/PAK3 interaction and membrane formation, and on touching any 
surface, the outer ELP-rich layers of the membrane adhere to the surface and immediately disassemble at the point of contact (Supplementary Fig. 12). We hypothesize that as the configuration of the ELP opens and more PAK3 fibres self-assemble, the ELP5/PAK3 membrane progressively accumulates elastic energy, which results in the formation of a prestressed viscoelastic gel layer that ruptures on contact with the external surfaces. The release of the stored elastic energy produces a stress that opens the membrane. This phenomenon may be similar to that reported by Sykes and co-workers ${ }^{34}$, in which local rupture of an actin membrane resulted in symmetry breaking and the release of elastic energy accumulated during the formation of the membrane ${ }^{35,36}$. In the system described by Sykes and co-workers, elastic stress resulted from the polymerization of a branched actin network ${ }^{34}$; however, in our case the stress may emerge from the PAK3-driven ELP5 opening and subsequent ELP5/PAK3 interactions within the membrane. A second process that could aid the opening of the membrane results from surface-tension gradients. Surface-tension measurements were conducted on ELP5 and PAK3 solutions. Both molecules decreased the surface tension, which suggests the possibility that PA molecules create a Marangoni-like effect to produce a tangential interfacial stress that could assist the membrane aperture. The ELP molecules at the air-water interface could enhance the membrane adhesion at the point of contact with the interface (Supplementary Fig. 13) 25,26.

Growth of the tubular structures can be triggered simply by displacing the interface. The interfacial area generated by this mechanical disturbance allows further ELP/PA interaction and membrane growth by self-assembly. Formation and growth of tubular structures by displacing the interface can be sustained for substantial periods of time, as long as sufficient amounts of ELP and PA molecules are present in solution. This hypothesis was confirmed by growing tubes using different concentrations of PAK3 or by injecting additional PAK3 solution into the inside of a formed tube. As expected, longer tubes were grown in both scenarios (Fig. 3e), which demonstrates that this stage of morphogenesis takes place through further recruitment of ELP and PA molecules from the solutions.

Non-equilibrium systems depend on a continuous flux and dissipation of energy to control interactions between many components and generate processes such as force transmission, motility, self-healing and morphogenesis ${ }^{32}$. The non-equilibrium self-assembly of peptide-based materials capable of responding to modifications in local chemical gradients has been reported ${ }^{37}$. Otto and co-workers have also demonstrated how additional energy input in the form of mechanical perturbations can influence self-assembly ${ }^{38}$. Our system responds to instabilities that arise from both gradients in chemical potential and mechanical perturbations. By controlling these stimuli, the system can be 'switched on' to access non-equilibrium on demand and be maintained in this state for substantial periods of time as the self-assembly is guided hierarchically and with a high spatiotemporal control.

Application in tissue engineering. ELP and PA molecules can be functionalized easily to create complex scaffolds. This potential was assessed by culturing primary mouse-adipose-derived stem cells (mADSCs) and human umbilical vein endothelial cells (hUVECs) on ELP5/PAK3 tubes for 21 days (Fig. 4). Both cell types were seeded onto the tubes in serum-free medium for four hours and serum-supplemented medium for up to 21 days (Fig. 4 and Supplementary Fig. 14). At four hours, cells were attached and already spread on the tubes (Fig. 4a,c). Throughout the culture, cells exhibited a higher preference for adhesion and spreading on the outer surface of the tubes compared with the inner surface (Fig. 4d and Supplementary Section 15), and also were observed to grow within the multilayers (Fig. 4c). This enhanced cellular adhesion may be related to the presence of the RGDS sequence in the ELP5 molecules on the outer surface of the tubes (Fig. 2a,b). SEM imaging showed little to no ECM presence around the cells that adhered on the inner surface of the tube, but a large and thick layer of ECM was observed around the cells that grew on the outer surface (Fig. 4c). Furthermore, metabolic activity was maintained for up to 21 days with a measured activity equivalent to cells seeded on tissue culture plastic (TCP), which confirms the viability of the cells that grow on the tubes (Fig. 4b). Functionalized ELPs have been used to create bioactive matrices for tissue engineering 39,40 and PA matrices have been moulded into tubes that support cell growth ${ }^{41}$. Our system provides a leap forward by enabling the fabrication of geometrically complex scaffolds solely by directed self-assembly and without the need for moulds or templates. In addition, hybrid structures that enable the use of proteins offer tunability and a higher level of scaffold complexity, versatility and functionality.

\section{Conclusion}

This work demonstrates the possibility of exploring supramolecular interactions between peptides and proteins and applying them in innovative ways to generate materials with novel properties. We designed and developed a dynamic self-assembly system that is capable of maintaining a controlled non-equilibrium state for substantial periods of time. The system emerges from a PA-driven opening of ELP molecules that co-assemble into a membrane with a distinctive multilayered architecture, and enables its morphogenesis into tubular structures with high spatiotemporal control. These tubular structures are robust and can be grown indefinitely as long as sufficient PA and ELP molecules are available. The building blocks, ELP5 and PAK3, are modular and can be modified easily to incorporate further functionality. We show that the system can be used to self-assemble bioactive tubular scaffolds that can support and guide the growth of mADSCs and hUVECs. Applications in tissue engineering and models for cell studies or drug screening, which require a higher biomimetic degree in recreating tubular structures, would particularly benefit from this approach.

\section{Methods}

All the materials and methods, including peptide synthesis and characterization, are included in the Supplementary Information.

Received 14 May 2015; accepted 18 August 2015; published online 28 September 2015

\section{References}

1. Sasai, Y. Cytosystems dynamics in self-organization of tissue architecture. Nature 493, 318-326 (2013).

2. Whitesides, G. M. \& Grzybowski, B. Self-assembly at all scales. Science 295, 2418-2421 (2002).

3. Silva, N. C. S. et al. Protein-based materials: from sources to innovative sustainable materials for biomedical applications. J. Mater. Chem. B 2 3715-3740 (2014).

4. Urry, D. W. et al. in Biotechnological Polymers: Medical, Pharmaceutical and Industrial Applications (ed. Gebelein, C. G.) 82-103 (Technomic, 1993).

5. Smits, F. C., Buddingh, B. C., van Eldijk, M. B. \& van Hest, J. C. Elastin-like polypeptide based nanoparticles: design rationale toward nanomedicine. Macromol. Biosci. 15, 36-51 (2015).

6. Almine, J. F. et al. Elastin-based materials. Chem. Soc. Rev. 39, 3371-3379 (2010).

7. Martin, L., Castro, E., Ribeiro, A., Alonso, M. \& Rodriguez-Cabello, J. C. Temperature-triggered self-assembly of elastin-like block co-recombinamers: the controlled formation of micelles and vesicles in an aqueous medium. Biomacromolecules 13, 293-298 (2012).

8. Rodriguez-Cabello, J. C., Prieto, S., Reguera, J., Arias, F. J. \& Ribeiro, A. Biofunctional design of elastin-like polymers for advanced applications in nanobiotechnology. J. Biomater. Sci. Polym. Ed. 18, 269-286 (2007).

9. Fletcher, J. M. et al. Self-assembling cages from coiled-coil peptide modules. Science 340, 595-599 (2013). 
10. Tang, T.-Y.et al. Fatty acid membrane assembly on coacervate microdroplets as a step towards a hybrid protocell model. Nature Chem. 6, 527-533 (2014).

11. Hartgerink, J. D., Beniash, E. \& Stupp, S. I. Self-assembly and mineralization of peptide-amphiphile nanofibers. Science 294, 1684-1688 (2001).

12. Zhang, S. et al. A self-assembly pathway to aligned monodomain gels. Nature Mater. 9, 594-601 (2010).

13. Glaab, F., Kellermeier, M., Kunz, W., Morallon, E. \& Garcia-Ruiz, J. M.

Formation and evolution of chemical gradients and potential differences across self-assembling inorganic membranes. Angew. Chem. Int. Ed. 51, 4317-4321 (2012).

14. Ritchie, C. et al. Spontaneous assembly and real-time growth of micrometre- scale tubular structures from polyoxometalate-based inorganic solids. Nature Chem. 1,47$52(2009)$.

15. Noorduin, W. L., Grinthal, A., Mahadevan, L. \& Aizenberg, J. Rationally designed complex, hierarchical microarchitectures. Science 340, 832-837 (2013).

16. Galland, R. et al. Fabrication of three-dimensional electrical connections by means of directed actin self-organization. Nature Mater. 12, 416-421 (2013).

17. Adler-Abramovich, L. et al. Self-assembled arrays of peptide nanotubes by vapour deposition. Nature Nanotech. 4, 849-854 (2009).

18. Capito, R. M., Azevedo, H. S., Velichko, Y. S., Mata, A. \& Stupp, S. I. Self-

assembly of large and small molecules into hierarchically ordered sacs and membranes. Science 319, 1812-1816(2008).

19. Ferreira, D. S., Marques, A. P., Reis, R. L. \& Azevedo, H. S. Hyaluronan and self- assembling peptides as building blocks to reconstruct the extracellular environment in skin tissue. Biomater. Sci. 1, 952-964 (2013).

20. Carvajal, D. et al. Physical properties of hierarchically ordered self-assembled planar and spherical membranes. Soft Matter 6, 1816-1823 (2010).

21. Chow, L. W. et al. A bioactive self-assembled membrane to promote angiogenesis. Biomaterials 32, 1574-1582 (2011).

22. Velichko, Y. S. et al. Electric field controlled self-assembly of hierarchically ordered membranes. Adv. Funct. Mater. 22, 369-377 (2012).

23. Carew, D. B., Channon, K. J., Manners, I. \& Woolfson, D. N. Polyelectrolite- surfactant nanocomposite membranes formed at liquid-liquid interface. Soft Matter 7 , 3475-3481(2011).

24. Urry, D. W. Physical chemistry of biological free energy transduction as demonstrated by elastin protein-based polymers. J. Phys. Chem. B 101, 11007-11028 (1997).

25. Miller, R. et al. Dynamics of protein and mixed protein/surfactant adsorption layers at the water/fluid interface. Adv. Colloid Interface Sci. 86, 39-82 (2000).

26. Kotsmar, Cs et al. Thermodynamics, adsorption kinetics and rheology of mixed protein-surfactant interfacial layers. Adv. Colloid Interface Sci. 150, 41-54 (2009).

27. Bitton, R. et al. Electrostatic control of structure in self-assembled membranes. Small 10, 500-505 (2014)

28. Boekhoven, J. et al. Alginate-peptide amphiphile core-shell microparticles as a targeted drug delivery system. RSC Adv. 5, 8753-8756 (2015).

29. Mertens, H. D. \& Svergun, D. I. Structural characterization of proteins and complexes using small-angle X-ray solution scattering. J. Struct. Biol. 172, 128-141 (2010).

30. Giehm, L., Oliveira, C. L., Christiansen, G., Pedersen, J. S. \& Otzen, D. E. SDS-induced fibrillation of alpha-synuclein: an alternative fibrillation pathway. J. Mol. Biol. 401, 115-133 (2010).

31. Andersen, K. K. et al. The role of decorated SDS micelles in sub-CMC protein denaturation and association. J. Mol. Biol. 391, 207-226 (2009).

32. Mann, S. Self-assembly and transformation of hybrid nano-objects and nanostructures under equilibrium and non-equilibrium conditions. Nature Mater. 8, 781-792 (2009).

33. Meinhardt, H. \& Gierer, A. Pattern formation by local self-activation and lateral inhibition. Bioessays 22, 753-760 (2000). 
34. Kawska, A. et al. How actin network dynamics control the onset of actin-based motility. Proc. Natl Acad. Sci. USA 109, 14440-14445 (2012).

35. Siton, O. et al. Cortactin releases the brakes in actin-based motility by enhancing WASP-VCA detachment from Arp2/3 branches. Curr. Biol. 21, 2092-2097 (2011).

36. Bernheim-Groswasser, A., Wiesner,S., Golsteyn, R. M.,Carlier, M.F.\& Sykes, C. The dynamics of actin-based motility depend on surface parameters. Nature 417,308311 (2002).

37. Hirst, A. R. et al. Biocatalytic induction of supramolecular order. Nature Chem.

$$
\text { 2, 1089-1094 (2010). }
$$

38. Carnall, J. M. et al. Mechanosensitive self-replication driven by self-organization. Science 327, 1502-1506 (2010)

39. Boccafoschi, F. et al. Human elastin polypeptides improve the biomechanical properties of three-dimensional matrices through the regulation of elastogenesis. $J$. Biomed. Mater. Res. A 103,1218-1230(2015).

40. Choi, S. K., Park, J. K., Lee, K. M., Lee, S. K. \& Jeon, W. B. Improved neural progenitor cell proliferation and differentiation on poly(lactide-co-glycolide) scaffolds coated with elastin-like polypeptide. J. Biomed. Mater. Res. B 101, 1329-1339 (2013)

41. McClendon, M. T. \& Stupp, S. I. Tubular hydrogels of circumferentially aligned nanofibers to encapsulate and orient vascular cells. Biomaterials $33,5713-5722$ (2012). 